\title{
An Initial Study into the Role of Teriparatide in Absent or Delayed Regenerate Formation during Distraction Osteogenesis: A Case Series
}

\author{
Bharath Patil $^{1}$, Rajeev Kansay ${ }^{2}$, Sandeep Gupta ${ }^{3}$, Anil Kapoor ${ }^{4}$, Anmol Sharma $^{5}$, Naveen Mittal ${ }^{6}$
}

\begin{abstract}
Purpose: Distraction osteogenesis has been used for post-traumatic segmental bone defects. Absent or delayed callus formation in the distraction gap can lead to significant morbidity and affect the clinical outcome. Experimental evidence in animal models has demonstrated that teriparatide enhances the consolidation of regenerate and also strengthens it. This study aimed to report our experience with recombinant teriparatide therapy for patients with regenerate insufficiency.

Materials and methods: Nine out of 43 patients undergoing limb lengthening using the limb reconstruction system (LRS) fixator were diagnosed with regenerate insufficiency. With informed consent, these patients received a therapeutic regime of $20 \mu \mathrm{g}$ of teriparatide administered subcutaneously once daily for a period of 3 months.

Results: The mean age in the sample was 40.22 years (SD 17.87). Regenerate insufficiency was diagnosed at a mean of 4.94 months (range 2.5-9 months) from surgery. Teriparatide injections were started at a mean of 6.94 months (range $4.5-11$ months) from surgery. The sites of regenerate insufficiency were tibia $(n=5)$ and femur $(n=4)$. Favourable radiographic progress in visibility of callus was seen at a mean duration of 9.4 weeks (range 8-12 weeks) from the initiation of teriparatide therapy. No systemic complications were encountered.

Conclusion: The initiation of teriparatide treatment as described in this study may be successful in triggering the osteogenic potential within poor regenerate and help in consolidation and avoid more invasive surgical procedures.

Keywords: Absent callus, Delayed callus, Distraction osteogenesis, Poor regenerate, Teriparatide.

Strategies in Trauma and Limb Reconstruction (2020): 10.5005/jp-journals-10080-1464
\end{abstract}

\section{INTRODUCTION}

Distraction osteogenesis (DO) is a standard of treatment for posttraumatic segmental bone defects. Various forms of external fixators have been used using the principle of DO with uniformly good results. ${ }^{1}$ The success of this treatment modality depends on adequate regenerate formation along with its consolidation. Despite best efforts, few patients have either delay in the regenerate formation and/or consolidation. This inadequacy of regenerate formation can complicate the treatment by prolonging the fixator time. ${ }^{2-4}$

Various techniques have been reported to salvage such a scenario. Slowing the rate of distraction, stopping distraction or the accordion manoeuvre are some of the mechanical ways of improving the regenerate. ${ }^{1,5-7}$ Pharmaceuticals have been tried, e.g., systemic administration of bisphosphonates, the local introduction of growth factors, such as, bone morphogenetic proteins, bone marrow cells. ${ }^{8-12}$

There have been animal studies that suggest that recombinant parathyroid hormone (PTH) not only enhances new bone formation but also helps in the consolidation of the poorly formed callus. There may be the prevention of fixator-related osteoporosis of the surrounding bone segments. ${ }^{13,14}$ This study aimed to report our experience using recombinant PTH therapy for patients with regenerate insufficiency midway through the lengthening process.

\section{Materials and Methods}

Forty-three patients underwent limb lengthening using a monorail fixator (Limb Reconstruction System, Orthofix SRL, Verona, Italy)

\footnotetext{
1,2,4 Department of Orthopaedics, Government Medical College and Hospital, Chandigarh, India

3,5 Department of Orthopaedics, Fortis Hospital, Mohali, Punjab, India ${ }^{6}$ Department of Orthopaedics, Shalby Hospital, Chandigarh, India
}

Corresponding Author: Sandeep Gupta, Department of Orthopaedics, Fortis Hospital, Mohali, Punjab, India, Phone: +91 9815933514, e-mail: sandy_tdh@yahoo.com

How to cite this article: Patil B, Kansay R, Gupta S, et al. An Initial Study into the Role of Teriparatide in Absent or Delayed Regenerate Formation during Distraction Osteogenesis: A Case Series. Strategies Trauma Limb Reconstr 2020;15(2):117-120.

Source of support: Nil

Conflict of interest: None

between 2016 and 2018. Nine patients were diagnosed with poor regenerate formation. In all the patients, regenerate was assessed by using plain radiographs and classified based on Fernandez Esteve grading: ${ }^{15}$

- Grade I-Empty space between two fragments without radiopacity.

- Grade II-Presence of cloud of bony callus.

- Grade III-Presence of periosteal bridge in at least one diaphyseal wall in every X-ray projection.

- Grade IV-Presence of periosteal bridge in both diaphyseal walls in every X-ray projection.

- Grade V-Structural callus is seen.

() The Author(s). 2020 Open Access This article is distributed under the terms of the Creative Commons Attribution 4.0 International License (https://creativecommons. org/licenses/by-nc/4.0/), which permits unrestricted use, distribution, and non-commercial reproduction in any medium, provided you give appropriate credit to the original author(s) and the source, provide a link to the Creative Commons license, and indicate if changes were made. The Creative Commons Public Domain Dedication waiver (http://creativecommons.org/publicdomain/zero/1.0/) applies to the data made available in this article, unless otherwise stated. 
The patients who were diagnosed with regenerate insufficiency or a stall in progression were advised to slow down the rate of distraction for 1 month. Eventually, distraction was stopped for 1 month and the regenerate then analysed again with fresh radiographs. Patients who did not show any improvement or progress in regenerate formation or maturity were started on daily subcutaneously administered teriparatide (twenty micrograms) as treatment. Radiographs were obtained every 2 weeks to monitor progress. This treatment was approved by the hospital ethics committee and informed consent was obtained from all the patients. The permission to review the cases was provided by the local ethics committee.

\section{Results}

The sample consisted of nine patients who were diagnosed with regenerate insufficiency. The demographic details are in Table 1.

Regenerate insufficiency was diagnosed at a mean of 4.94 months (range 2.5-9) from surgery. Teriparatide injections were started at a mean of 6.94 months (range 4.5-11) from surgery. All patients were given teriparatide therapy for duration of 3 months. Favourable radiographic progress in the callus was seen at a mean of 9.4 weeks (range 8-12 weeks) after the initiation of teriparatide therapy. Of the nine patients, four were found to have grade I regenerate and five had grade II regenerate using the Fernandez Esteve grading. ${ }^{15}$ There was a statistically significant difference in mean time taken to achieve visibility of radiographic progress ( $p$ value 0.035 ): This was 10.35 weeks in patients with grade I callus formation and 8.6 weeks in those with grade II callus. All nine

Table 1: Demographic data of patients

\begin{tabular}{ll}
\hline Characteristic & \\
\hline Mean age (years, SD) & $40.22(17.87)$ \\
Male & 9 \\
Female & 0 \\
Mean size of the defect (cm, SD) & $9.17(4.4)$ \\
Site of non-union & \\
Tibia & 5 \\
Femur & 4 \\
\hline
\end{tabular}

patients were followed up for a minimum of 1 year (range 1-3) and all the patients went on to have full bone consolidation in the distraction gap at the end of follow-up.

\section{Case Description}

A 50-year-old man diagnosed with an infected non-union of the right tibia, who underwent limb lengthening with composite fixation using a locked plate simultaneously with a monorail fixator $^{16}$ was diagnosed with grade II regenerate insufficiency at 4 months postoperatively. The patient was advised to slow down distraction for 1 month and the distraction was stopped for 1 month. Follow-up X-rays 2 months later did not show any progress in the consolidation of the regenerate. With informed consent, injections of teriparatide were started at a dose of $20 \mu \mathrm{g}$ subcutaneously once daily. Radiographic progress in regenerate consolidation was visible at 8 weeks after therapy initiation (Fig. 1). The patient subsequently underwent autologous bone grafting and medial plate augmentation at the docking site. X-rays at the final follow-up 6 months later showed complete consolidation of the regenerate with the union at the docking site. The patient was walking full weight-bearing without any support (Fig. 2).

A 25-year-old man diagnosed with an infected non-union of the left femur, who underwent limb lengthening with composite fixation using a locked plate simultaneously with a monorail fixator was diagnosed with grade II regenerate insufficiency at 3 months postoperatively. After diagnosing the patient with regenerate insufficiency, teriparatide therapy was started and X-rays were done 10 weeks after therapy initiation showed visible radiographic progress in regenerate consolidation (Fig. 3). X-rays at the final follow-up showed complete consolidation of the regenerate (Fig. 4).

\section{Discussion}

Distraction osteogenesis involves mechanical stretching from a lowenergy osteotomy to encourage the formation of new bone in the gap created between the gradually separated skeletal fragments. Poor regenerate formation leads to significant morbidity as the fixator needs to be kept in place for an extended period of time until the bone is completely consolidated. This has an unfavourable

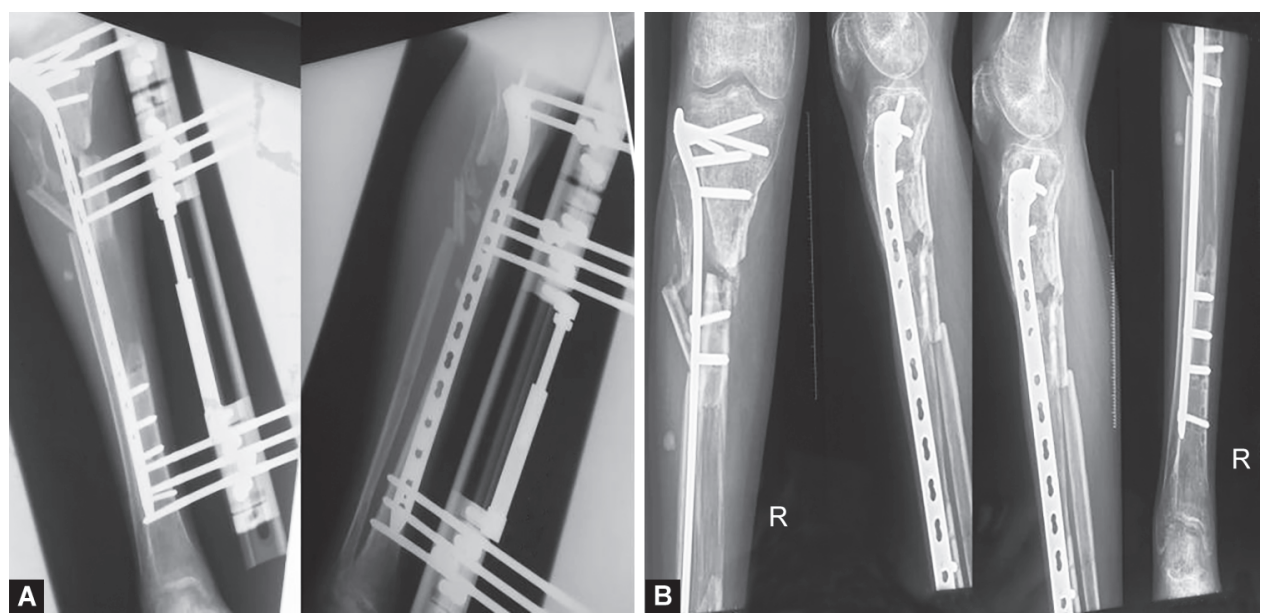

Figs $1 \mathrm{~A}$ and B: Antero-posterior and lateral radiographic projections of a patient with an infected non-union of the right tibia. This was treated by resection and limb lengthening using composite fixation of a locked plate simultaneously with a monorail (limb reconstruction system) fixator. The regenerate was diagnosed showing grade II insufficiency at 4 months postoperatively and further X-rays showed visible progress in regenerate consolidation at 8 weeks after therapy initiation 
psychological impact and can be associated with increased pin site infections, persistent pain and increased risk of osteopenia. ${ }^{2-4}$

Various modes for rescue in such situations, e.g., systemic administration of pharmaceutical agents, such as, bisphosphonates, local exogenous administration of growth factors including bone morphogenetic proteins or bone marrow cells, and the use of externally applied low-intensity pulsed ultrasound and pulsed electromagnetic fields have yielded varying results. ${ }^{8-12}$ Kiely et al. ${ }^{17}$ reported that six of the seven children treated with parenteral bisphosphonates for the poor quality of regenerate in DO healed eventually without further intervention. No side-effects related to bisphosphonate therapy were reported. But, given the prolonged half-life of bisphosphonates and their influence on bone growth and remodelling, as well as other systemic effects, the use of these agents warrants further study.

There have been animal experimental studies on the use of teriparatide for poor regenerate formation. ${ }^{13,14}$ Seebach et al. ${ }^{14}$ studied the effects of intermittent doses of PTH on rats undergoing femur lengthening with an external fixator and found that, within 20 days after starting treatment with teriparatide (1-34), there was an increase in ultimate load, stiffness, total regenerate
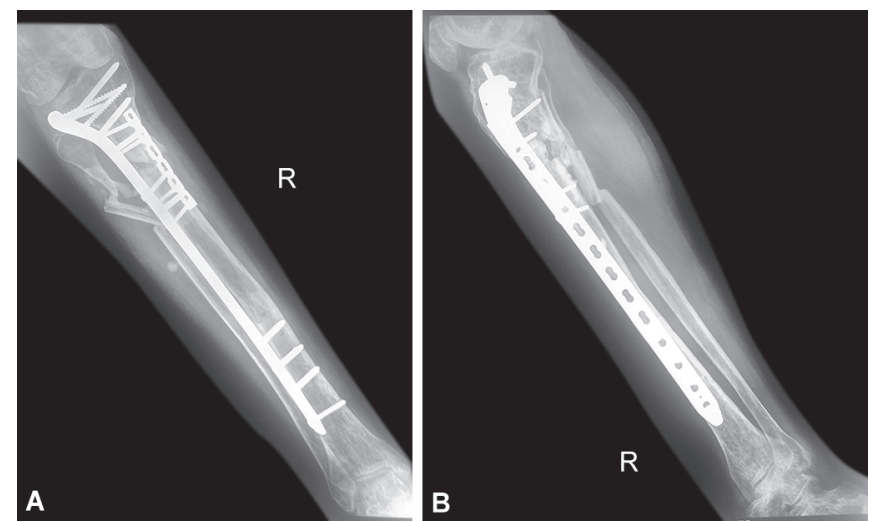

Figs 2A and B: Antero-posterior and lateral X-rays at final follow-up showing complete consolidation of the regenerate with union at docking site callus volume, callus bone mineral content and histological bone density compared to those untreated. They concluded PTH (1-34) might become useful in shortening the consolidation time after DO in humans. Similarly, Aleksyniene et al. ${ }^{18}$ conducted a study in rabbits treated with PTH $25 \mu \mathrm{g} / \mathrm{kg} /$ day for 30 days during DO and concluded that treatment with PTH during DO resulted in substantially higher mineralised tissue volume, mineral content and bending strength. They suggested that treatment with PTH may benefit new bone formation during DO and could form a basis for the clinical application of this therapy in humans. Multiple studies have proposed that PTH stimulates osteogenic differentiation of mesenchymal stem cells and hence leads to increased mineralisation of the regenerate and a reduction of the amount of fibrous tissue. ${ }^{13,19}$ Wagner et al. ${ }^{20}$ conducted a randomised control study on 16 patients undergoing DO in whom some were given teriparatide injections for a period of 8 weeks during the consolidation phase and found that teriparatide treatment during the consolidation phase of DO doubled the mineralisation rate of the regenerate when compared with no treatment.

All the above-mentioned studies led us to believe that intermittently administered PTH enhances bone mineral content in the regenerate and helps in the consolidation of poorly formed regenerate. In our study, we found radiographic improvement in the regenerate quality was visible as early as 8 weeks after the initiation of teriparatide therapy. The treatment was given for a total period of 3 months in all the patients and at the end of follow-up, all the nine patients had full consolidation. Although regenerate formation and consolidation may be influenced by the type of fixation used for limb lengthening (circular llizarov fixators provide better three-dimensional stability compared to a monorail fixator), we used composite fixation of a locked plate along with a rail fixator where previously published results were comparable to that of circular fixators. ${ }^{16}$

This treatment was well tolerated by all the patients. None reported any adverse effects. There are concerns over teriparatideinduced osteosarcoma but this was associated with long-term usage and high doses in the Fischer 344 (F344) rats. No such effect was detected in other animal models or human trials. ${ }^{21}$ Another
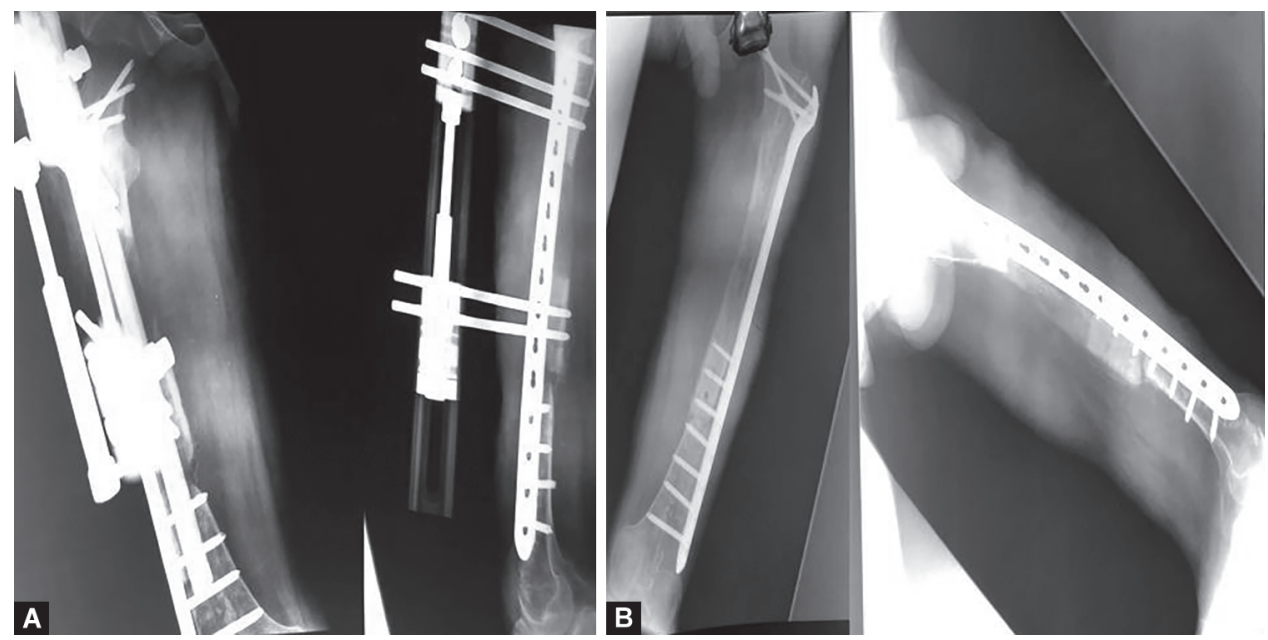

Figs $3 \mathrm{~A}$ and B: Antero-posterior and lateral X-rays of a 25-year-old male patient diagnosed with an infected non-union of the left femur. This was treated by resection and limb lengthening using composite fixation of a locked plate simultaneously with a monorail (LRS) fixator. A diagnosis of grade II regenerate insufficiency was made at 3 months postoperatively and further X-rays of femur showed visible progress in regenerate consolidation at 10 weeks after therapy initiation 

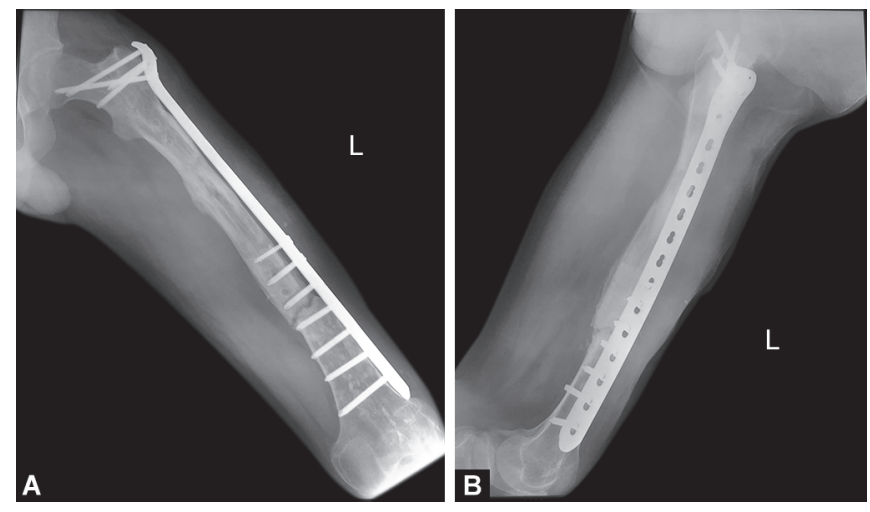

Figs $4 A$ and $B$ : $X$-rays at final follow-up showing complete consolidation of the regenerate

limiting factor with this therapy is the higher cost for treatment and potential poor patient compliance as it needs to be administered subcutaneously daily for a period of 3 months.

This study has limitations. It is essentially a pilot study in the form of a small case series without a control group. The conclusions cannot be over-reaching and the results have to be taken in the context of the exposure to several sources of bias. However, the initial results here should prompt the investigation of teriparatide in a controlled study. Delayed or absent regenerate bone formation is a complication during limb lengthening and further investigation of potentially helpful pharmaceutical therapies is warranted.

\section{SUMmARY}

We conclude that the initiation of the teriparatide regimen may be successful in triggering the osteogenic potential within the poor regenerate and promote consolidation by avoiding more invasive surgical procedures.

\section{Disclosure}

None of the authors received payments or services, either directly or indirectly (i.e., via his/her institution), from a third party in support of any aspect of this work.

\section{Consent to Participate}

Written informed consent was obtained from all the patients participating in the study and the use of data and demographic details and radiological images for publication purposes.

\section{Ethical Approval}

All procedures performed in studies involving human participants were in accordance with the ethical standards of the institutional and/or national research committee and with the 1964 Helsinki declaration and its later amendments or comparable ethical standards. Informed written consent was obtained from all the patients included in the study.

\section{References}

1. Ilizarov G. Clinical application of the tension-stress effect for limb lengthening. Clin Orthopaed Relat Res 1990;250(250):8-26. DOI: 10.1097/00003086-199001000-00003.
2. Velazquez RJ, Bell DF, Armstrong PF, et al. Complications of use of the Ilizarov technique in the correction of limb deformities in children. J Bone Joint Surg Am 1993;75(8):1148-1156. DOI: 10.2106/00004623199308000-00004.

3. Garcia-Cimbrelo E, Olsen B, Ruiz-Yague M, et al. Ilizarov technique: results and difficulties. Clin Orthop Relat Res 1992(283):116-123.

4. Eldridge JC, Bell DF. Problems with substantial limb lengthening. Orthop Clin North Am 1991;22(4):625-631.

5. Claes $L$, Augat $P$, Schorlemmer $S$, et al. Temporary distraction and compression of a diaphyseal osteotomy accelerates bone healing. J Orthop Res 2008;26(6):772-777. DOI: 10.1002/jor.20588.

6. Mora R. Nonunion of the long bones: diagnosis and treatment with compression-distraction techniques. Milan, Italy: Springer; 2006.

7. Hamdy RC, Rendon JS, Tabrizian M. Distraction osteogenesis and its challenges in bone regeneration. Bone Regeneration Tal H, ed. ch. 8 pp. 177-204. InTech, Rijeka, Croatia 2012.

8. Makhdom AM, Hamd RC. The role of growth factors on acceleration of bone regeneration during distraction osteogenesis. Tissue Eng Part B Rev 2013;19(5):442-453. DOI: 10.1089/ten.teb.2012.0717.

9. Aronson J. Experimental and clinical experience with distraction osteogenesis,. Cleft Palate Craniofac J 1994;31(6):473-482. DOI: 10.1597/1545-1569_1994_031_0473_eacewd_2.3.co_2.

10. Sabharwal S. Enhancement of bone formation during distraction osteogenesis: pediatric applications. J Am Acad Orthopaed Surg 2011;19(2):101-111. DOI: 10.5435/00124635-201102000-00005.

11. Gebauer D, Correll J. Pulsed low-intensity ultrasound: anew salvage procedure for delayed unions and non unions after leg lengthening in children. J Pediat Orthopaed 2005;25(6):750-754. DOI: 10.1097/01. bpo.0000173245.12184.7e.

12. Eyres KS, Saleh M, Kanis JA. Effect of pulsed electromagnetic fields on bone formation and bone loss during limb lengthening. Bone 1996;18(6):505-509. DOI: 10.1016/8756-3282(96)00070-1.

13. Ye B, Li Y, Zhu S, et al. Effects of intermittent low-dose parathyroid hormone treatment on rapid mandibular distraction osteogenesis in rabbits. J Oral Maxillofac Surg 2017;75(8):1722-1731. DOI: 10.1016/ j.joms.2017.04.010.

14. Seebach C, Skripitz R, Andreassen TT, et al. Intermittent parathyroid hormone (1-34) enhances mechanical strength and density of new bone after distraction osteogenesis in rats. J Orthop Res 2004;22(3):472-478. DOI: 10.1016/j.orthres.2003.08.018.

15. Catagni M. Imaging techniques: the radiographic classification of bone regenerate during distraction. Operative Principles of Ilizarov Maiocchi AB, Aronson J, ed., London: Williams \& Wilkins; 1991. pp. 53-57.

16. Gupta S, Malhotra A, Mittal N, et al. The management of infected nonunion of tibia with a segmental defect using simultaneous fixation with a monorail fixator and a locked plate. Bone Joint J 2018;100-B(8):1094-1099. DOI: 10.1302/0301-620X.100B8.BJJ-20171442.R1.

17. Kiely $\mathrm{P}$, Ward K, Bellemore $\mathrm{CM}$, et al. Bisphosphonate rescue in distraction osteogenesis: a case series. J Pediatr Orthop 2007;27(4):467-471. DOI: 10.1097/01.bpb.0000271326.41363.d1.

18. Aleksyniene $\mathrm{R}$, Thomsen JS, Eckardt $\mathrm{H}$, et al. Parathyroid hormone PTH(1-34) increases the volume, mineral content, and mechanical properties of regenerated mineralizing tissue after distraction osteogenesis in rabbits. Acta Orthop 2009;80(6):716-723. DOI: 10.3109/17453670903350032.

19. Sheyn D, Cohn Yakubovich D, Kallai I, et al. PTH promotes allograft integration in a calvarial bone defect. Mol Pharm 2013;10(12):4462. DOI: $10.1021 / \mathrm{mp} 400292 \mathrm{p}$.

20. Wagner F, Vach W, Augat $P$, et al. Daily subcutaneous teriparatide injection increased bone mineral density of newly formed bone after tibia distraction osteogenesis, a randomized study. Injury 2019;50(8):1478-1482. DOI: 10.1016/j.injury.2019.06.001.

21. Subbiah V, Madsen VS, Raymond AK, et al. Of mice and men: divergent risks of teriparatide-induced osteosarcoma. Osteoporos Int 2010;21(6):1041-1045. DOI: 10.1007/s00198-009-1004-0. 